\title{
SELF-DIFFUSION OF SPHERES IN A CONCENTRATED SUSPENSION
}

\author{
$\mathrm{C} \mathrm{W} \mathrm{J}$ BEENAKKER and $\mathrm{P}$ MAZUR
}

Instituut-Lorentz, Ryksuniversitelt te Letden, Nieuwsteeg 18, 2311 SB Letden, The Netherlands

Received 1 March 1983

We calculate the concentration-dependence of the short-tıme self-diffusion coefficient $\boldsymbol{D}_{\mathrm{s}}$ for spherical particles in suspension Our analysis is valid up to high densities and fully takes into account the many-body hydrodynamic interactions between an arbitrary number of spheres. The importance of these many-body interactions can be inferred from our calculation of the second virial coefficient of $\boldsymbol{D}_{\mathrm{s}}$.

\section{Introduction}

It is well-known that properties of a suspension of particles in a fluid (e.g., diffusion, sedimentation, viscosity) are concentration-dependent, due to direct interparticle interactions and due to a coupling of their motion via the fluid. This coupling is called hydrodynamic interaction and is the subject of our investigation.

The influence of hydrodynamic interactions on properties of suspensions can be studied conveniently by analyzing the concentration-dependence of the so-called short-time self-diffusion coefficient of uncharged spherical particles in suspension. This quantity (which we denote by $\boldsymbol{D}_{\mathrm{s}}$ ) describes diffusion of a single "tracer" particle on a time-scale, over which the spatial configuration of the particles is essentially constant $t^{1,2}$ ). If the mobilities of the spheres are known-as a function of their positions - it is possible to calculate $\boldsymbol{D}_{\mathrm{s}}$ by means of a generalized Einstein relation ${ }^{3}$ ), which relates $\boldsymbol{D}_{\mathrm{s}}$ to an average of these mobilities over all the configurations of the spheres. Experimentally the short-time self-diffusion coefficient can be determined from dynamic light-scattering studies: the initial decay of the auto-correlation function of the scattered field at large values of the scattering vector yields values for $\boldsymbol{D}_{\mathrm{s}}{ }^{4}$ ).

If the suspension is sufficiently dilute we can assume the hydrodynamic couplings to be pairwise additive, i.e. we need to consider only two-body hydrodynamic interactions. Most theoretical treatments of properties of suspensions are restricted to this low-density regime*: the linear density corrections

* An exception is formed by Muthukumar et al., who included many-body hydrodynamic interactions in their analysis of Darcy-flow (cf. ref. 5 and the references therein). 
to the values at infinite dilution of $\boldsymbol{D}_{\mathrm{s}}$ and of the bulk-diffusion coefficient were calculated by Batchelor ${ }^{3}$ ) and by Felderhof ${ }^{6}$ ) and Jones ${ }^{7}$ ) Batchelor used generalızed Einstein relations for these coefficients, while Felderhof and Jones based their analysıs on a Fokker-Planck equation in the many-partıcle coordınate space Their results were equivalent For the case of bulk-diffusion the value of this first virial coefficient has been confirmed by experiments ${ }^{8}$ )

Recent theoretical results on many-body hydrodynamic interactions $s^{9}$ ) (see also ref 10) enabled us to extend the analysis of Batchelor ${ }^{3}$ ) to include second-order density corrections As we reported in ref 11, we could conclude from our calculations that three-body hydrodynamic interactions may not be neglected if the suspension is not dilute (see in this connection also ref 12) At stıll higher densities one will have to take into account the full many-body hydrodynamic interaction Moreover, an expansion in the density (a "virial expansion") is not appropriate in this high-density regime

In the present work we present a theory for the concentration dependence of the short-time self-diffusion coefficient $\boldsymbol{D}_{\mathrm{s}}$, which is valid up to high densities and which fully takes into account the many-body hydrodynamic interactions between an arbitrary number of spheres In section 2 we summarize the expressions for the many-sphere mobilities ${ }^{9}$ ) and derive a few formulae for later use, by means of an Einstein relation ${ }^{3}$ ) we can express $\boldsymbol{D}_{\mathrm{s}}$ in terms of these mobilities, cf eqs (32) and (3 16)

In the latter equation the contributions due to hydrodynamic interactions between clusters of $2,3,4,5, \quad$ spheres are formally resummed In the sections 5, 6 and 7 we evaluate $D_{\mathrm{s}}$ as the average of an expansion in powers of the fluctuation in the concentration of the suspended particles The zeroth order approximation (no density fluctuations) can be called an effective medium or continuum theory for the self-diffusion coefficient, which can then be expressed in terms of an effective viscosity $\dagger$ We will include in our calculation of $\boldsymbol{D}_{\mathrm{s}}$ the lowest (second) order correction due to fluctuations in the concentration From our numerical results we can conclude that this fluctuation expansion can describe the concentration dependence of $\boldsymbol{D}_{\mathrm{s}}$ reasonably well up to high densities

Eq (3 2) on the other hand is a surtable starting point for a virial expansion in the volume fraction $\phi$ Detarls on the calculation of the second virial coefficient of $\boldsymbol{D}_{\mathrm{s}}\left(\mathrm{e}\right.$ e the coefficient of the term of order $\phi^{2}$ ) are given in section 4 (cf ref 11 where also the bulk-diffusion coefficient was calculated to the same order in $\phi)$

Finally in section 8 we discuss our results and compare them with experiments ${ }^{14}$ )

$\dagger$ The Clausius-Mossott1 formula for the dielectric constant can similarly be regarded as the zeroth order result from a fluctuation expansion Bedeaux and Mazur ${ }^{13}$ ) have investigated in this context the deviations due to density fluctuations 


\section{Mobilities}

Consider $N$ equal-sized spherical particles with radius $a$ and position-vectors $\boldsymbol{R}_{\imath}$ $(i=1,2, \ldots N)$, moving in an incompressible fluid with viscosity $\eta$, which is otherwise at rest. We describe the motion of the fluid by the linear quasi-static Stokes equation, supplemented by stick boundary conditions at the surfaces of the spheres. The velocity $\boldsymbol{u}_{i}$ of sphere $i$ can be expressed as a linear combination of the forces $\boldsymbol{K}_{j}$, exerted by the fluid on each of the spheres $j$

$$
\boldsymbol{u}_{i}=-\sum_{j=1}^{N} \boldsymbol{\mu}_{y} \cdot \boldsymbol{K}_{j}, \quad i=1,2, \ldots N .
$$

The mobility tensors $\boldsymbol{\mu}_{i j}$ depend on the configuration of the $N$ spheres; a term in $\boldsymbol{\mu}_{i j}$ which depends on the positions of $s$ spheres is said to reflect $s$-body hydrodynamic interactions. In eq. (2.1) we have assumed that the fluid exerts no torque on the spheres, i.e. each sphere can rotate freely.

The general expression for the mobilities, as derived in ref. 9, has the structure of an infinite series of reflections or scatterings from the spheres,

$$
\begin{aligned}
& (6 \pi \eta a) \boldsymbol{\mu}_{i j}=1 \delta_{\eta j}+\boldsymbol{A}_{! t}^{(1,1)}+\sum_{s=1}^{\infty} \sum_{m_{1}=2}^{\infty} \sum_{m_{2}=2}^{\infty} \ldots \sum_{m_{s}=2}^{\infty} \sum_{\substack{j_{1}=1 \\
J_{1} \neq 1}}^{N} \sum_{\substack{j_{2} \\
j_{2} \neq J_{1}}}^{N} \ldots \\
& \times \sum_{\substack{J_{s}=1 \\
J_{\uparrow} \neq J_{s}-1, j}}^{N} \boldsymbol{A}_{l_{1}}^{\left(1, m_{1}\right)} \odot \boldsymbol{B}^{\left(m_{1}, m_{1}\right)-1} \odot \boldsymbol{A}_{J_{1} J_{2}}^{\left(m_{1}, m_{2}\right)} \odot \ldots \odot \boldsymbol{B}^{\left(m_{s}, m_{s}\right)-1} \odot \boldsymbol{A}_{J_{s} J}^{\left(m_{s}, 1\right)}
\end{aligned}
$$

and is given as a sum of products of tensors called connectors. The connector $\boldsymbol{A}_{l j}^{(n, m)}(i \neq j)$ is a tensor of rank $n+m$, which characterizes a hydrodynamic interaction between a force multipole of order $n$ on sphere $i$ and a multipole of order $m$ on sphere $j$. This connector is a function of $\boldsymbol{R}_{i j} \equiv \boldsymbol{R}_{j}-\boldsymbol{R}_{\imath}$ of order $\left(a /\left|\boldsymbol{R}_{l y}\right|\right)^{n+m-1}$ and hence, for large separation of the spheres, low values of $n$ and $m$ dominate. By definition these connectors are zero for $i=j$. The tensor $\boldsymbol{B}^{(m, m)-1}$ is a generalized inverse of a tensor $\boldsymbol{B}^{(m, m)}$ of rank $2 m$, which does not depend on the positions of the spheres. The notation $\boldsymbol{A}_{i j}^{(n, m)} \odot \boldsymbol{B}^{(m, m)^{-1}}$ prescribes an $m$-fold contraction, with the convention that the last index of the first tensor is contracted with the first index of the second tensor, etc.

The general expressions for the connectors are (cf. ref. 9)

$$
\begin{aligned}
& \boldsymbol{A}_{n !}^{(n, m)}=0, \\
& \boldsymbol{A}_{l j}^{(n, m)}=\int \mathrm{d} \boldsymbol{r} \int \mathrm{d} \boldsymbol{r}^{\prime} \delta\left(\boldsymbol{R}_{\imath}-\boldsymbol{r}\right) \delta\left(\boldsymbol{R}_{J}-\boldsymbol{r}^{\prime}\right) \boldsymbol{A}^{(n, m)}\left(\boldsymbol{r}^{\prime}-\boldsymbol{r}\right), \quad i \neq j, \quad\left|\boldsymbol{R}_{l}\right|>2 a,
\end{aligned}
$$


with the connector field $\boldsymbol{A}^{(n m)}(\boldsymbol{r})$ given by

$$
\begin{aligned}
\boldsymbol{A}^{(n m)}(\boldsymbol{r})= & \left(\frac{4}{3} \pi a^{3}\right) \frac{9}{2}(2 n-1) "(2 m-1)^{n \prime}(1)^{m-n}(2 \pi)^{-3} \int \mathrm{d} \boldsymbol{k} \mathrm{e}^{-1 \boldsymbol{k} r}(a k)^{-2} \\
& \times\left(\frac{\partial^{n-1}}{\partial(a \boldsymbol{k})^{n-1}} \frac{\sin (a k)}{a k}\right)(\boldsymbol{1}-\hat{\boldsymbol{k}} \hat{k})\left(\frac{\hat{\partial}^{m-1}}{\partial(a \boldsymbol{k})^{m-1}} \frac{\sin (a k)}{a k}\right)
\end{aligned}
$$

The tensor $\boldsymbol{B}^{(2)^{-1}}$ is given by*

$$
B^{(22)} \cdot=-\frac{10}{9} \Delta^{(22)}
$$

The tensor $\boldsymbol{B}^{(m m)}$ for $m \geqslant 3$ is defined, in terms of the connector field (2 5), as

$$
\boldsymbol{B}^{(m m)}=-\boldsymbol{A}^{(m m)}(\boldsymbol{r}=0), \quad m \geqslant 3
$$

The inverse of this tensor is evaluated explicitly below

In the above equations $(2 n-1)^{\prime \prime}=135 \quad(2 n-3)(2 n-1), \quad k=|k|$, $\hat{\boldsymbol{k}}=\boldsymbol{k} / \boldsymbol{k}$ The notation $\widetilde{\boldsymbol{b}}^{p}$ denotes an irreducible tensor of rank $p, 1 \mathrm{e}$, a tensor traceless and symmetric in any pair of its indices, constructed from a $p$-fold ordered product of the vector $\boldsymbol{b}$ (in the present context $\boldsymbol{b}$ stands for $\partial / \partial(a \boldsymbol{k})$ ) For $p=1,2,3$ one has (see e g ref 15 )

$$
\begin{aligned}
& \vec{b}_{\alpha}=b_{\alpha}, \quad \vec{b}_{\alpha} b_{\beta}=b_{\alpha} b_{\beta}-\frac{1}{3} \delta_{\alpha \beta} b^{2}, \\
& \overparen{b_{\alpha} b_{\beta} b_{\gamma}}=b_{\alpha} b_{\beta} b_{y}-\frac{1}{5}\left(\delta_{\alpha \beta} b_{\gamma}+\delta_{\alpha \gamma} b_{\beta}+\delta_{\beta \gamma} b_{\alpha}\right) b^{2}
\end{aligned}
$$

The tensor $\Delta^{(2)}$ used in eq (26) belongs to a class of tensors $\Delta^{(n n)}$ of rank $2 n$, which project out the irreducible part of a tensor of rank $n$

$$
\Delta^{(n n)} \bigcirc b^{n}=b^{n} \bigcirc \Delta^{(n n)}=\overparen{b}^{n}
$$

For $n=1,2$ we have ${ }^{15}$ )

$$
\begin{aligned}
& \Delta_{\alpha \beta}^{(1)}=\{1\}_{\alpha \beta}=\delta_{\alpha \beta}, \\
& \Delta_{\alpha \beta \gamma \delta}^{(22)}=\frac{1}{2} \delta_{\alpha \delta} \delta_{\beta \gamma}+\frac{1}{2} \delta_{\alpha \gamma} \delta_{\beta \delta}-\frac{1}{3} \delta_{\alpha \beta} \delta_{\gamma \delta}
\end{aligned}
$$

The general expression for the mobilities as a function of the positions of the spheres (2 2) constitutes an expansion in the inverse interparticle distance $1 / R$ An explicit evaluation up to and including terms of order $(1 / R)^{7}$ can be found in ref 9

Eqs (2 3)-(2 10) define in principle all the quantities appearing in the expression (2 2) for the mobilities For later use it is convenient to rewrite the connector field (25) (from which connectors are formed according to eqs (24) and (2 7)) in a

\footnotetext{
* The tensor $\boldsymbol{B}^{(22)} 1$ defined here corresponds to $\boldsymbol{B}^{(2525)}$ ' in ref 9
} 
somewhat different form, using the identity

$$
\frac{\overparen{\partial^{p}}}{\partial \boldsymbol{k}^{p}} \frac{\sin k}{k}=\widehat{\boldsymbol{k}^{p}}(-1)^{p}\left(\frac{\pi}{2 k}\right)^{1 / 2} J_{p+1 / 2}(k), \quad p=0,1,2, \ldots,
$$

where $J_{p+1 / 2}$ is the Besselfunction of order $p+1 / 2$. Eq. (2.11) follows from the definition $^{16}$ )

$$
J_{p+1 / 2}(k)=(-1)^{p}\left(\frac{2 k}{\pi}\right)^{1 / 2} k^{p}\left(k^{-1} \mathrm{~d} / \mathrm{d} k\right)^{p} \frac{\sin k}{k}
$$

and the relation ${ }^{17}$ ) (which can be proven by induction)

$$
\frac{\partial^{p}}{\partial \boldsymbol{k}^{p}} f(k)=\widetilde{\boldsymbol{k}^{p}}\left(k^{-1} \mathrm{~d} / \mathrm{d} k\right)^{p} f(k) .
$$

If we now define a Fourier-transformed connector field

$$
\boldsymbol{A}^{(n, m)}(\boldsymbol{k})=\int \mathrm{d} \boldsymbol{r} \mathrm{e}^{\mathrm{ik} \cdot \boldsymbol{r}} \boldsymbol{A}^{(n, m)}(\boldsymbol{r})
$$

we have, in view of eqs. (2.5) and (2.11),

$$
\begin{aligned}
\boldsymbol{A}^{(n, m)}(\boldsymbol{k})= & \left(\frac{4}{3} \pi a^{3}\right) \frac{9}{2}(2 n-1) ! !(2 m-1) ! !(-i)^{m-n} \frac{\pi}{2}(a k)^{-3} \\
& \times J_{n-1 / 2}(a k) J_{m-1 / 2}(a k) \widehat{\boldsymbol{k}^{n-1}}(1-\hat{\boldsymbol{k}} \hat{\boldsymbol{k}}) \hat{\boldsymbol{k}^{m-1}}
\end{aligned}
$$

The above relation may be used to evaluate the tensor $\boldsymbol{B}^{(m, m)}$ explicitly for $m \geqslant 3$, since (cf. eq. (2.7))

$$
\boldsymbol{B}^{(m, m)}=-(2 \pi)^{-3} \int k^{2} \mathrm{~d} k \int \mathrm{d} \hat{\boldsymbol{k}} \boldsymbol{A}^{(m, m)}(\boldsymbol{k}), \quad m \geqslant 3 .
$$

The scalar part of the above integration may be evaluated with help of the formula (ref. 16, p. 679)

$$
\int_{0}^{\infty} \mathrm{d} k k^{-1} J_{m-1 / 2}^{2}(k)=(2 m-1)^{-1}
$$

Using, for the angular integration in eq. (2.16), the results given in appendix A (see eq. (A.9)), we find the explicit expression

$$
\begin{aligned}
\boldsymbol{B}^{(m, m)}= & -\frac{3}{2}(m-1) !(2 m-3) ! !\left(\boldsymbol{\Delta}^{(m-1, \mathrm{ld}, m-1)}-\frac{m}{2 m+1} \boldsymbol{\Delta}^{(m, m)}\right. \\
& \left.-\frac{m-1}{2 m-1} \boldsymbol{\Delta}^{(m-1, m-1)} \odot^{m-2} \boldsymbol{\Delta}^{(m-1, m-1)}\right), \quad m \geqslant 3,
\end{aligned}
$$


where the symbol $\odot^{l}$ denotes an $l$-fold contraction. The tensor $\Delta^{(m-1, d, m-1)}$ is a tensor of rank $2 m$ with elements

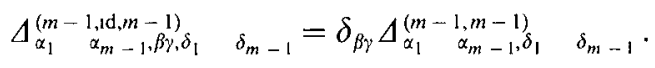

This tensor acts as a unit tensor when contracted with a tensor $\boldsymbol{T}^{(m)}$ of rank $m$, which is irreducible (traceless and symmetric) in its first $m-1$ indices*

$$
\boldsymbol{A}^{(m-1 \mathrm{~d} \mathrm{~d}, m-1)} \odot \boldsymbol{T}^{(m)}=\boldsymbol{T}^{(m)} .
$$

The tensor $\boldsymbol{B}^{(m, m)^{-1}}(m \geqslant 3)$ appearing in eq. (2.2) is the generalized inverse of $\boldsymbol{B}^{(m, m)}$ in the space of tensors of rank $m$ which are irreducible in their first $m-1$ indices. It is therefore determined by the equation

$$
\boldsymbol{B}^{(m, m)-1} \odot \boldsymbol{B}^{(m, m)}=\boldsymbol{A}^{(m-1, \mathrm{dd}, m-1)}, \quad m \geqslant 3 .
$$

The result

$$
\begin{aligned}
\boldsymbol{B}^{(m, m)-1}= & -\left[\frac{3}{2}(m-1) !(2 m-3) ! !\right]^{-1}\left(\boldsymbol{\Delta}^{(m-1, \mathrm{dd}, \mathrm{m}-1)}+\frac{m}{m+1} \boldsymbol{\Delta}^{(m, m)}\right. \\
& \left.+\frac{m-1}{m-2} \frac{2 m-3}{2 m-1} \boldsymbol{\Delta}^{(m-1, m-1)} \odot^{m-2} \boldsymbol{\Delta}^{(m-1, m-1)}\right), \quad m \geqslant 3
\end{aligned}
$$

may - with help of the formulae (A.3)-(A.5) in appendix A-be checked by substitution in eq. (2.21).

We recall that $\boldsymbol{B}^{(2,2)^{-1}}$ is defined in eq. (2.6).

\section{Self-diffusion}

The short-time self-diffusion coefficient $\boldsymbol{D}_{\mathrm{s}}$ is related to the mobilities discussed in section 2 by a generalized Einstein relation ${ }^{3}$ )

$$
\boldsymbol{D}_{\mathrm{s}}=k_{\mathrm{B}} T \frac{1}{N}\left\langle\sum_{\imath=1}^{N} \boldsymbol{\mu}_{u}\right\rangle
$$

where $\langle\ldots\rangle$ denotes an average over all configurations of the $N$ spheres inside a volume $V$. We denote the temperature and Boltzmann's constant by $T$ and $k_{\mathrm{B}}$, respectively. The short-time self-diffusion coefficient $\boldsymbol{D}_{\mathrm{s}}$ describes the diffusion of a single "tracer" particle, over distances small compared to the interparticle separation (see in this connection the discussion in ref. 2). Combining eqs. (2.2) and (3.1) we obtain (see also eq. (2.3))

* Notice that $\boldsymbol{A}_{y}^{(n m)}$ is by definition irreducible in its first $n-1$ and last $m-1$ indices 


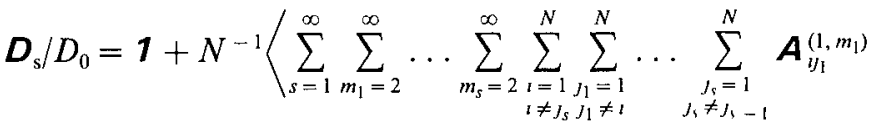

$$
\begin{aligned}
& \left.\odot \boldsymbol{B}^{\left(m_{1}, m_{1}\right)^{-1}} \odot \boldsymbol{A}_{J_{1 / 2}}^{\left(m_{1}, m_{2}\right)} \odot \ldots \odot \boldsymbol{B}^{\left(m_{s}, m_{s}\right)-1} \odot \boldsymbol{A}_{s_{s}}^{\left(m_{s}, 1\right)}\right\rangle,
\end{aligned}
$$

where we have defined

$$
D_{0}=k_{\mathrm{B}} T(6 \pi \eta a)^{-1} .
$$

Thus for isolated spherical particles $D_{\mathrm{s}}=D_{0} \mathbf{1}$, the familiar Stokes-Einstein result.

Eq. (3.2) will be the starting point for the virial expansion of $\boldsymbol{D}_{\mathrm{s}}$, evaluated up to second order in section 4 . In order to study also the behaviour of $\boldsymbol{D}_{\mathrm{s}}$ at higher densities, we will cast this equation in a different form, which permits a formal resummation. We first redefine the connector field in the following way

$$
\tilde{\boldsymbol{A}}^{(n, m)}(\boldsymbol{r})=\boldsymbol{A}^{(n, m)}(\boldsymbol{r}) \quad \text { if } \boldsymbol{r} \neq 0, \quad \tilde{\boldsymbol{A}}^{(n, m)}(\boldsymbol{r}=0)=0 .
$$

If we now use definition (2.4), eq. (3.2) becomes

$$
\begin{aligned}
\boldsymbol{D}_{\mathrm{s}} / D_{0}= & \boldsymbol{1}+N^{-1} \sum_{s=1}^{\infty} \sum_{m_{1}=2}^{\infty} \ldots \sum_{m_{\mathrm{s}}=2}^{\infty} \int \mathrm{d} \boldsymbol{r}_{0} \int \mathrm{d} \boldsymbol{r}_{1} \ldots \int \mathrm{d} \boldsymbol{r}_{s} \\
& \times\left\langle\sum_{l=1}^{N} \delta\left(\boldsymbol{R}_{t}-\boldsymbol{r}_{0}\right) \sum_{J_{1}=1}^{N} \delta\left(\boldsymbol{R}_{\boldsymbol{f}_{1}}-\boldsymbol{r}_{1}\right) \ldots \sum_{J_{s}=1}^{N} \delta\left(\boldsymbol{R}_{J_{s}}-\boldsymbol{r}_{s}\right)\right. \\
& \left.\times \tilde{\boldsymbol{A}}^{\left(1, m_{1}\right)}\left(\boldsymbol{r}_{1}-\boldsymbol{r}_{0}\right) \odot \boldsymbol{B}^{\left(m_{1}, m_{1}\right)-1} \odot \ldots \odot \tilde{\boldsymbol{A}}^{\left(m_{s}, 1\right)}\left(\boldsymbol{r}_{0}-\boldsymbol{r}_{s}\right)\right\rangle .
\end{aligned}
$$

Note that the introduction of the modified connector field $\tilde{\boldsymbol{A}}^{(n, m)}(\boldsymbol{r})$ enabled us to perform the summations over the particle indices without restriction. Due to homogeneity of the suspension, the integrand in eq. (3.5) is invariant under a translation of the particle position vectors over $\boldsymbol{r}_{0}$. After a change of integration variables eq. (3.5) takes the form

$$
\begin{aligned}
\boldsymbol{D}_{\mathrm{s}} / D_{0}= & \boldsymbol{1}+n_{0}^{-1} \sum_{s=1}^{\infty} \sum_{m_{1}=2}^{\infty} \ldots \sum_{m_{\mathrm{s}}=2}^{\infty} \int \mathrm{d} \boldsymbol{r}_{1} \int \mathrm{d} \boldsymbol{r}_{2} \ldots \int \mathrm{d} \boldsymbol{r}_{s} \\
& \times\left\langle n(\boldsymbol{r}=0) \tilde{\boldsymbol{A}}^{\left(1, m_{1}\right)}\left(\boldsymbol{r}_{1}\right) n\left(\boldsymbol{r}_{1}\right) \odot \boldsymbol{B}^{\left(m_{1}, m_{1}\right)-1} \odot \tilde{\boldsymbol{A}}^{\left(m_{1}, m_{2}\right)}\left(\boldsymbol{r}_{2}-\boldsymbol{r}_{1}\right)\right. \\
& \left.\times n\left(\boldsymbol{r}_{2}\right) \odot \ldots \odot \boldsymbol{B}^{\left(m_{s}, m_{s}\right)-1} \odot \tilde{\boldsymbol{A}}^{\left(m_{s}, 1\right)}\left(-\boldsymbol{r}_{s}\right)\right\rangle,
\end{aligned}
$$

where the microscopic density field, with average $n_{0}=N / V$, is given by

$$
n(\boldsymbol{r})=\sum_{i=1}^{N} \delta\left(\boldsymbol{R}_{\boldsymbol{t}}-\boldsymbol{r}\right)
$$

Eq. (3.6) may alternatively be written in operator notation

$$
\begin{aligned}
\boldsymbol{D}_{\mathrm{s}} / D_{0}= & \mathbf{1}+n_{0}^{-1} \sum_{s=1}^{\infty} \sum_{m_{1}=2}^{\infty} \ldots \sum_{m_{s}=2}^{\infty}\left\langle\left\{ n \tilde{\boldsymbol{A}}^{\left(1, m_{1}\right)} n \odot \boldsymbol{B}^{\left(m_{1}, m_{1}\right)^{-1}}\right.\right. \\
& \left.\left.\odot \tilde{\boldsymbol{A}}^{\left(m_{1}, m_{2}\right)} n \odot \ldots \odot \boldsymbol{B}^{\left(m_{1}, m_{s}\right)-1} \odot \tilde{\boldsymbol{A}}^{\left(m_{1}, 1\right)}\right\}(0 \mid 0)\right\rangle,
\end{aligned}
$$


where $n$ and $\tilde{\boldsymbol{A}}^{(n, m)}$ (written without argument) are linear integral operators with kernels

$$
\begin{aligned}
& n\left(\boldsymbol{r} \mid \boldsymbol{r}^{\prime}\right)=n(\boldsymbol{r}) \delta\left(\boldsymbol{r}^{\prime}-\boldsymbol{r}\right), \\
& \tilde{\boldsymbol{A}}^{(n, m)}\left(\boldsymbol{r} \mid \boldsymbol{r}^{\prime}\right)=\tilde{\boldsymbol{A}}^{(n, m)}\left(\boldsymbol{r}^{\prime}-\boldsymbol{r}\right) .
\end{aligned}
$$

We see that in $\boldsymbol{r}$-representation $n$ is a diagonal operator and $\tilde{\boldsymbol{A}}^{(n, m)}$ a convolution operator. The notation $\{\ldots\}(0 \mid 0)$ prescribes an evaluation of the kernel of the operator between braces at $\boldsymbol{r}=\boldsymbol{r}^{\prime}=0$.

Next we define matrices $\mathscr{A}$ and $\mathscr{B}^{-1}$ with elements

$$
\begin{aligned}
& \{\mathscr{A}\}_{n, m}=\tilde{\boldsymbol{A}}^{(n, m)}, \\
& \left\{\mathscr{B}^{-1}\right\}_{n, m}=\delta_{n m} \boldsymbol{B}^{(m, m)-1}
\end{aligned}
$$

and projection operators $P$ and $Q=1-P$

$$
\{P\}_{n, m}=\delta_{n 1} \delta_{m 1}, \quad\{Q\}_{n, m}=\delta_{n m}-\delta_{n 1} \delta_{m 1} .
$$

With these notations we may write e.g., ${ }^{*}$

$$
\sum_{m=2}^{\infty} \tilde{\boldsymbol{A}}^{(1, m)} \odot \boldsymbol{B}^{(m, m)-1} \odot \tilde{\boldsymbol{A}}^{(m, 1)}=P \mathscr{A} Q \mathscr{B}^{-1} \mathscr{A} P
$$

and eq. (3.8) takes the form

$$
\boldsymbol{D}_{\mathrm{s}} / D_{0}=1+n_{0}^{-1} \sum_{s=1}^{\infty} P\left\langle\left\{n \mathscr{A}\left(n Q \mathscr{B}^{-1} \mathscr{A}\right)^{s}\right\}(0 \mid 0)\right\rangle P .
$$

This equation can formally be resummed to yield

$$
\boldsymbol{D}_{\mathrm{s}} / D_{0}=1+n_{0}^{-1} P\left\langle\left\{n \mathscr{A}\left(1-n Q \mathscr{B}^{-1} \mathscr{A}\right)^{-1}\right\}(0 \mid 0)\right\rangle P,
$$

where we have used the fact that, in view of definition (3.4),

$$
P\langle\{n \mathscr{A}\}(0 \mid 0)\rangle P=n_{0} \tilde{A}^{(1,1)}(r=0)=0 .
$$

We remark that it is possible to derive eq. (3.16) algebraically from eqs. (5.2)-(5.5) of ref. 9, in a way which does not require a resummation. Eq. (3.16) - which contains the full hydrodynamic interaction of the $N$ spheres - will be the starting point for the expansion of $\boldsymbol{D}_{\mathrm{s}}$ in correlations of density fluctuations, performed in section 5 .

\section{The virial expansion}

For a dilute suspension it is appropriate to express the short-time self-diffusion coefficient $\boldsymbol{D}_{\mathrm{s}}$ as a power series in the density $n_{0}$ (a so-called virial expansion). We

\footnotetext{
* To be more precise: the r.h.s. of eq. (3.14) is a matrix with the 1.h.s. as the only non-zero element.
} 
shall evaluate this series up to and including terms of second order in the density. Up to this order we need consider only two- and three-body hydrodynamic interactions, since the probability that a given particle has $s$ neighbours is of order $n_{0}^{s}$. The contributions to the virial expansion of $\boldsymbol{D}_{\mathrm{s}}$ originating from two- and three-body hydrodynamic interactions are discussed separately in subsections 4.1 and 4.2 , respectively.

\subsection{Two-sphere contributions}

A restriction of $\mu_{l j}$ to terms which depend on the positions of at most two spheres has the following expansion in powers of $1 / R^{189}$ )

$$
\begin{gathered}
(6 \pi \eta a) \mu_{i l}(\text { two-spheres })=1+\sum_{k \neq i}\left(-\frac{15}{4}\right)\left(a / R_{k k}\right)^{4} \hat{r}_{l k} \hat{r}_{l k} \\
+\sum_{k \neq 1} \frac{1}{16}\left(a / R_{l k}\right)^{6}\left(105 \hat{r}_{l k} \hat{r}_{l k}-171\right)+\mathcal{O}(a / R)^{8} .
\end{gathered}
$$

Here the vector $\boldsymbol{R}_{t k}=\boldsymbol{R}_{k}-\boldsymbol{R}_{t}$ has magnitude $R_{t k}=\left|\boldsymbol{R}_{t k}\right|$ and direction $\hat{\boldsymbol{r}}_{t k}=\boldsymbol{R}_{t k} / \boldsymbol{R}_{t k}$. Substitution of eq. (4.1) in eq. (3.2) yields for the two sphere contributions to $\boldsymbol{D}_{\mathrm{s}}$

$$
\begin{aligned}
& \boldsymbol{D}_{\mathrm{s}} / D_{0} \text { (two-spheres) }=1+n_{0} \int \mathrm{d} \boldsymbol{R} g(R) \\
& \quad \times\left(-\frac{15}{4}(a / R)^{4} \hat{\boldsymbol{r}} \hat{\mathbf{r}}+\frac{1}{16}(a / R)^{6}(105 \hat{\boldsymbol{r}} \hat{r}-171)\right),
\end{aligned}
$$

where $g(R)$ denotes the pair-distribution function for two spheres separated by $R$. Up to order $n_{0}$ we have (see e.g. ref. 19)

$$
g(R)= \begin{cases}0, & \text { if } R<2 a, \\ 1+\frac{4}{3} \pi a^{3} n_{0}\left(8-3 R / a+\frac{1}{16}(R / a)^{3}\right), & \text { if } 2 a \leqslant R \leqslant 4 a, \\ 1, & \text { if } R>4 a .\end{cases}
$$

An elementary integration gives the required first and second order density corrections to $D_{0}$ due to two-body hydrodynamic interactions

$$
\left.D_{\text {s }} \text { (two-spheres }\right)=D_{0} 1\left[1-1.73 \phi-0.93 \phi^{2}+\mathcal{O}\left(\phi^{3}\right)\right],
$$

where $\phi$ is the partial volume or volume fraction of the spheres

$$
\phi=\frac{4}{3} \pi a^{3} n_{0} .
$$

\subsection{Three-sphere contributions}

Of the three-sphere terms in the expansion of $\boldsymbol{\mu}_{n}$ in powers of $1 / R$ we have 
retained the dominant one (which is of order $R^{-7}$ ), evaluated in ref. 9,

$$
\begin{aligned}
& (6 \pi \eta a) \boldsymbol{\mu}_{l}(\text { three-spheres })=\sum_{k \neq l} \sum_{l \neq l, k}-\frac{75}{16}\left(a / R_{l k}\right)^{2}\left(a / R_{k l}\right)^{3}\left(a / R_{l l}\right)^{2} \\
& \times \hat{r}_{l k} \hat{r}_{l l}\left(\left(1-3 \xi_{k}^{2}\right)\left(1-3 \xi_{l}^{2}\right)+6 \xi_{k}^{2} \xi_{l}^{2}+6 \xi_{l} \xi_{k} \xi_{l}\right)+\mathcal{O}(a / R)^{9},
\end{aligned}
$$

where $\xi_{l}=\hat{\boldsymbol{r}}_{i k} \cdot \hat{\boldsymbol{r}}_{l l}, \xi_{k}=\hat{\boldsymbol{r}}_{k l} \cdot \hat{\boldsymbol{r}}_{k l}, \xi_{l}=\hat{\boldsymbol{r}}_{l l} \cdot \hat{\boldsymbol{r}}_{l k}$ are direction-cosines. The three-sphere contribution to $\boldsymbol{D}_{\mathrm{s}}$ is obtained by averaging eq. (4.6) with the three-sphere distribution function $g\left(R_{12}, R_{13}, R_{23}\right)$, given in lowest order by

$$
g\left(R_{12}, R_{13}, R_{23}\right)= \begin{cases}0, & \text { if } R_{12}<2 a \text { or } R_{13}<2 a \text { or } R_{23}<2 a \\ 1, & \text { elsewise }\end{cases}
$$

After three trivial angular integrations, we are left with a three-dimensional integral over a complicated domain, determined by eq. (4.7). This integral was evaluated numerically using Monte-Carlo techniques*. The resulting three-sphere contribution to $\boldsymbol{D}_{\mathrm{s}}$ is

$$
D_{s}(\text { three-spheres })=D_{0} 1\left[1.80 \phi^{2}+\mathcal{O}\left(\phi^{3}\right)\right] .
$$

If we add eqs. (4.4) and (4.8) we obtain the virial expansion of $\boldsymbol{D}_{\mathrm{s}}$ up to second order in the density (communicated by us in ref. 11)

$$
\left.D_{\text {s }} \text { (two- and three-spheres }\right)=D_{0} 1\left[1-1.73 \phi+0.88 \phi^{2}+\mathcal{O}\left(\phi^{3}\right)\right] \text {. }
$$

The term of order $\phi$ is well- $\left.-\mathrm{known}^{3,7}\right)$. Batchelor ${ }^{3}$ ) used exact expressions for the two-sphere mobility tensors and found $-1.83 \phi$ for the correction of order $\phi$. Comparison with eq. (4.9) shows that the terms of order $R^{-8}$ and higher neglected in eq. (4.1) are not very important. Concerning the three-sphere contributions neglected in eq. (4.6) (of order $R^{-9}$ and higher), we can say the following: a calculation of the contribution to $\boldsymbol{D}_{\mathrm{s}}$ due to one of the three-sphere mobility terms of order $R^{-9}$ gives $\dagger$ about $1 \%$ of the value in eq. (4.8), which results from the only term of order $R^{-7}$.

We defer a discussion of our result (4.9) to section 8 .

* Use was made of the adaptive stratified Monte-Carlo integration program RIWIAD ${ }^{20}$ )

$\dagger$ Using the notation of ref. 9 we found that the sequence of connectors

$$
\begin{aligned}
& \boldsymbol{G}^{(125)}\left(\boldsymbol{R}_{1}\right) \boldsymbol{B}^{(2 s 25)-1} \boldsymbol{H}^{(2 s 25)}\left(\boldsymbol{R}_{12}\right) B^{(2 s 25)-1} \boldsymbol{G}^{(2 s 1)}\left(-\boldsymbol{R}_{2}\right) \\
& \text { contributes }-0.016 \phi^{2} \text { to } D_{\mathrm{s}} / D_{0} .
\end{aligned}
$$




\section{The fluctuation expansion}

The fluctuations in the microscopic density field are defined by

$$
n(\boldsymbol{r})=n_{0}+\delta n(\boldsymbol{r})
$$

The average $\langle\delta n(\boldsymbol{r})\rangle$ equals zero by definition, while $\left.{ }^{21}\right)$

$$
\left\langle\delta n(\boldsymbol{r}) \delta n\left(\boldsymbol{r}^{\prime}\right)\right\rangle=n_{0} \delta\left(\boldsymbol{r}^{\prime}-\boldsymbol{r}\right)+n_{0}^{2}\left[g\left(\left|\boldsymbol{r}^{\prime}-\boldsymbol{r}\right|\right)-1\right],
$$

with $g(r)$ the pair distribution function.

Our aim is to expand the expression between braces in eq. (3.16) in powers of $\delta n$. This can be done most conveniently by using first the identity ( $A$ is an arbitrary operator)

$$
\left[1-\left(n_{0}+\delta n\right) A\right]^{-1}=\left(1-n_{0} A\right)^{-1}\left[1-\delta n A\left(1-n_{0} A\right)^{-1}\right]^{-1} .
$$

Substitution of (5.1) in eq. (3.16) gives, with the aid of (5.3), the alternative expression for $\boldsymbol{D}_{\mathrm{s}}$

$$
\boldsymbol{D}_{\mathrm{s}} / D_{0}=1+n_{0}^{-1} P\left\langle\left\{n \mathscr{A}_{n_{0}}\left[1-\delta n Q \mathscr{B}^{-1} \mathscr{A}_{n_{0}}\right]^{-1}\right\}(0 \mid 0)\right\rangle P,
$$

where the renormalized matrix of connectors $\mathscr{A}_{n_{0}}$ is defined as

$$
\mathscr{A}_{n_{0}} \equiv \mathscr{A}\left[1-n_{0} Q \mathscr{B}^{-1} \mathscr{A}\right]^{-1} .
$$

This renormalization accounts for the fact that fluctuations in the concentration of the spheres interact hydrodynamically via the suspension rather than through the pure fluid.

If we expand the expression between braces in eq. (5.4) in powers of $\delta n$, we obtain an expansion of $\boldsymbol{D}_{\mathrm{s}}$ in correlations of density fluctuations of higher and higher order (a "fluctuation expansion")

$$
\boldsymbol{D}_{\mathrm{s}}=\boldsymbol{D}_{\mathrm{s}}^{(0)}+\boldsymbol{D}_{\mathrm{s}}^{(2)}+\ldots
$$

where $\boldsymbol{D}_{\mathrm{s}}^{(p)}$ contains terms of order $\left\langle(\delta n)^{p}\right\rangle$.

The zeroth order term $\boldsymbol{D}_{\mathrm{s}}^{(0)}$ is given by

$$
\boldsymbol{D}_{\mathrm{s}}^{(0)} / D_{0}=\mathbf{1}+\boldsymbol{A}_{n_{0}}^{(1,1)}(\boldsymbol{r}=0),
$$

where the renormalized connector field $\boldsymbol{A}_{n_{0}}^{(n, m)}(\boldsymbol{r})$ is the kernel of the convolution operator $\boldsymbol{A}_{n_{0}}^{(n, m)}$, which in turn is an element of the matrix $\mathscr{A}_{n_{0}}$

$$
\boldsymbol{A}_{n_{0}}^{(n, m)}=\left\{\mathscr{A}_{n_{0}}\right\}_{n, m} .
$$

The renormalized connector field will be evaluated in the next section; an explicit expression for $\boldsymbol{D}_{s}^{(0)}$ is given in section 7 .

We will include in our calculation of $\boldsymbol{D}_{\mathrm{s}}$ the lowest order correction to $\boldsymbol{D}_{\mathrm{s}}^{(0)}$ due to fluctuations in the concentration of the suspended particles. This correction $\boldsymbol{D}_{\mathrm{s}}^{(2)}$ 
results from terms of order $\left\langle(\delta n)^{2}\right\rangle$ in eq. (5.4) and is given by

$$
\begin{aligned}
\boldsymbol{D}_{\mathrm{s}}^{(2)} / D_{0}= & n_{0}^{-1} P\left\langle\left\{\delta n \mathscr{A}_{n_{0}} \delta n Q \mathscr{B}^{-1} \mathscr{A}_{n_{0}}\right.\right. \\
& \left.\left.+n_{0} \mathscr{A}_{n_{0}} \delta n Q \mathscr{B}^{-1} \mathscr{A}_{n_{0}} \delta n Q \mathscr{B}^{-1} \mathscr{A}_{n_{0}}\right\}(0 \mid 0)\right\rangle P,
\end{aligned}
$$

or, written out explicitly,

$$
\begin{aligned}
\boldsymbol{D}_{i}^{(2)} / \boldsymbol{D}_{0}= & \sum_{m=2}^{\infty} \boldsymbol{A}_{n_{0}}^{(1, m)}(\boldsymbol{r}=0) \odot \boldsymbol{B}^{(m, m)-1} \odot \boldsymbol{A}_{n_{0}}^{(m, 1)}(\boldsymbol{r}=0) \\
& +\sum_{m=2}^{\infty} n_{0} \int \mathrm{d} \boldsymbol{r} \boldsymbol{A}_{n_{0}}^{(1, m)}(\boldsymbol{r}) \odot \boldsymbol{B}^{(m, m)-1} \odot \boldsymbol{A}_{n_{0}}^{(m, 1)}(-\boldsymbol{r})[g(\boldsymbol{r})-1] \\
& +\sum_{m=2}^{\infty} \sum_{k=2}^{\infty} n_{0} \int \mathrm{d} \boldsymbol{r} \boldsymbol{A}_{n_{0}}^{(1, m)}(\boldsymbol{r}) \odot \boldsymbol{B}^{(m, m)^{-1}} \odot \boldsymbol{A}_{n_{0}}^{(m, k)}(0) \\
& \odot \boldsymbol{B}^{(k, k)^{-1}} \odot \boldsymbol{A}_{n_{0}}^{(k, 1)}(-\boldsymbol{r}) \\
& +\sum_{m=2}^{\infty} \sum_{k=2}^{\infty} n_{0}^{2} \int \mathrm{d} \boldsymbol{r} \int \mathrm{d} \boldsymbol{r}^{\prime} \boldsymbol{A}_{n_{0}}^{(1, m)}(\boldsymbol{r}) \odot \boldsymbol{B}^{(m, m)-1} \odot \boldsymbol{A}_{n_{0}}^{(m, k)}\left(\boldsymbol{r}^{\prime}-\boldsymbol{r}\right) \\
& \odot \boldsymbol{B}^{(k, k)-1} \odot \boldsymbol{A}_{n_{0}}^{(k, 1)}\left(-\boldsymbol{r}^{\prime}\right)\left[g\left(\left|\boldsymbol{r}^{\prime}-\boldsymbol{r}\right|\right)-1\right] .
\end{aligned}
$$

Use has also been made of eq. (5.2). The contributions to $\boldsymbol{D}_{\mathrm{s}}^{(2)}$ result from pair-correlations (the terms containing $g(r)-1)$ and from self-correlations which would also be present in the hypothetical case of penetrable spheres.

\section{Evaluation of the renormalized connectors}

According to eqs. (5.5) and (5.8) the renormalized connector field $\boldsymbol{A}_{n_{0}}^{(n, m)}(\boldsymbol{r})$ is formally given by

$$
\begin{aligned}
\boldsymbol{A}_{n_{0}}^{(n, m)}(\boldsymbol{r})= & \tilde{\boldsymbol{A}}^{(n, m)}(\boldsymbol{r})+\sum_{s=1}^{\infty} \sum_{m_{1}=2}^{\infty} \ldots \sum_{m_{\mathrm{s}}=2}^{\infty} n_{0}^{s} \int \mathrm{d} \boldsymbol{r}_{1} \ldots \int \mathrm{d} \boldsymbol{r}_{s} \tilde{\boldsymbol{A}}^{\left(n, m_{1}\right)}\left(\boldsymbol{r}_{1}\right) \\
& \odot \boldsymbol{B}^{\left(m_{1}, m_{1}\right)-1} \odot \tilde{\boldsymbol{A}}^{\left(m_{1}, m_{2}\right)}\left(\boldsymbol{r}_{2}-\boldsymbol{r}_{1}\right) \odot \ldots \odot \boldsymbol{B}^{\left(m_{\mathrm{s}}, m_{\mathrm{s}}\right)-1} \odot \tilde{\boldsymbol{A}}^{\left(m_{\mathrm{s}}, m_{1}\right)}\left(\boldsymbol{r}-\boldsymbol{r}_{\mathrm{s}}\right),
\end{aligned}
$$

cf. also the definitions (3.10)-(3.13). We observe that we may replace $\tilde{\boldsymbol{A}}^{(n, m)}(\boldsymbol{r})$ under the integral in eq. (6.1) by $\boldsymbol{A}^{(n, m)}(\boldsymbol{r})$, since these two connector fields differ by a finite amount in a single point only, cf. eq. (3.4). Hence, in terms of the Fourier-transformed connector field defined in eq. (2.14), eq. (6.1) takes the form

$$
\begin{aligned}
\boldsymbol{A}_{n_{0}}^{(n, m)}(\boldsymbol{r})= & \tilde{\boldsymbol{A}}^{n, m)}(\boldsymbol{r})+\sum_{s=1}^{\infty} \sum_{m_{1}=2}^{\infty} \ldots \sum_{m_{\mathrm{r}}=2}^{\infty} n_{0}^{s}(2 \pi)^{-3} \int \mathrm{d} \boldsymbol{k} \mathrm{e}^{-1 \boldsymbol{k} \cdot \boldsymbol{r}} \boldsymbol{A}^{\left(n, m_{1}\right)}(\boldsymbol{k}) \\
& \odot \boldsymbol{B}^{\left(m_{1}, m_{1}\right)^{-1}} \odot \boldsymbol{A}^{\left(m_{1}, m_{2}\right)}(\boldsymbol{k}) \odot \ldots \odot \boldsymbol{B}^{\left(m_{s}, m_{s}\right)^{-1}} \odot \boldsymbol{A}^{\left(m_{s}, m^{\prime}\right)}(\boldsymbol{k}) .
\end{aligned}
$$


To proceed we make use of the formula (proven in appendix B)

$$
\begin{gathered}
n_{0} \boldsymbol{A}^{(n, p)}(\boldsymbol{k}) \odot \boldsymbol{B}^{(p, p)-1} \odot \boldsymbol{A}^{(p, m)}(\boldsymbol{k})=-\phi \boldsymbol{A}^{(n, m)}(\boldsymbol{k}) \frac{9}{2} \epsilon_{p} \\
\times(2 p-1)^{2} \frac{\pi}{2}(a k)^{-3} J_{p-1 / 2}^{2}(a k), \quad p \geqslant 2,
\end{gathered}
$$

with the definition

$$
\epsilon_{2}=5 / 9, \quad \epsilon_{p}=1 \quad(p \geqslant 3) .
$$

The volume fraction $\phi$ is defined in eq. (4.5). Using well-known formulae for Bessel functions (cf. appendix $\mathrm{C}$ ), we can analytically perform a summation over $p$ in eq. (6.3)

$$
\sum_{p=2}^{\infty} n_{0} \boldsymbol{A}^{(n, p)}(\boldsymbol{k}) \odot \boldsymbol{B}^{(p, p)-1} \odot \boldsymbol{A}^{(p, m)}(\boldsymbol{k})=-\phi S(a k) \boldsymbol{A}^{(n, m)}(\boldsymbol{k}),
$$

where the function $S(x)$ is given by

$$
\begin{aligned}
S(x)= & \frac{9}{2}\left[\operatorname{Si}(2 x) x^{-1}+\frac{1}{2} \cos (2 x) x^{-2}+\frac{1}{4} \sin (2 x) x^{-3}\right. \\
& \left.-(\sin x)^{2} x^{-4}-4(\sin x-x \cos x)^{2} x^{-6}\right] .
\end{aligned}
$$

Here the sine-integral $\mathrm{Si}(2 x)$ is defined by

$$
\operatorname{Si}(2 x)=\int_{0}^{2 x} \sin t / t \mathrm{~d} t .
$$

For small values of $x, S(x)$ behaves as

$$
S(x)=5 / 2+\mathcal{O}\left(x^{2}\right) .
$$

With the aid of formula (6.5) we can resum the formal expansion (6.2) to yield the required expression for the renormalized connector field

$$
\boldsymbol{A}_{n_{0}}^{(n, m)}(\boldsymbol{r})=\tilde{\boldsymbol{A}}^{(n, m)}(\boldsymbol{r})-(2 \pi)^{-3} \int \mathrm{d} \boldsymbol{k} \mathrm{e}^{-i k \cdot r} \boldsymbol{A}^{(n, m)}(\boldsymbol{k}) \phi S(a k)[1+\phi S(a k)]^{-1} .
$$

We remark that as a consequence of the expansion (6.8), we have for $r$ large

$$
\boldsymbol{A}_{n_{0}}^{(n, m)}(\boldsymbol{r})=\boldsymbol{A}^{(n, m)}(\boldsymbol{r})\left(1+\frac{5}{2} \phi\right)^{-1}\left[1+\mathcal{O}(a / r)^{2}\right] .
$$

We thus see that the range of a renormalized connector is the same as the range of an unrenormalized one, or, in other words, the hydrodynamic interaction is not screened in the effective medium.

For the fluctuation expansion we need in particular the value $\boldsymbol{A}_{n_{0}}^{(n, m)}(\boldsymbol{r}=0)$ (cf. 
eqs. (5.7) and (5.10)). Using, for the angular integration in eq. (6.9), the results (A.9)-(A.11) from appendix $\mathrm{A}$ we find

$$
\begin{aligned}
& \boldsymbol{A}_{m_{0}}^{(1)}(\boldsymbol{r}=0)=-\mathbf{1} \int_{0}^{\infty} \mathrm{d} k k^{-1} J_{1 / 2}^{2}(k) \phi S(k)[1+\phi S(k)]^{-1} \\
& \boldsymbol{B}^{(m m)^{-1}} \odot \boldsymbol{A}_{n_{0}}^{(m)}(\boldsymbol{r}=0) \odot \boldsymbol{B}^{(m, m)-1} \\
& =\boldsymbol{B}^{(m, m)-1}(2 m-1) \int_{0}^{\infty} \mathrm{d} k k^{-1} J_{m-1 / 2}^{2}(k) \phi S(k)[1+\phi S(k)]^{-1}, \quad m \geqslant 2,(6) \\
& \boldsymbol{A}_{n_{0}}^{(m, m+2)}(\boldsymbol{r}=0)=\boldsymbol{A}_{n_{0}}^{(m+2 m)}(\boldsymbol{r}=0)=-\frac{3}{2}(m+1) !(2 m-1) ! ! \boldsymbol{A}^{(m+1 m+1)} \\
& \quad \times \int_{0}^{\infty} \mathrm{d} k k^{-1} J_{m-1 / 2}(k) J_{m+3 / 2}(k) \phi S(k)[1+\phi S(k)]^{-1} \\
& \boldsymbol{A}_{r_{0}}^{(n m)}(\boldsymbol{r}=0)=0 \text { if } n \neq m \text { and } n \neq m \pm 2
\end{aligned}
$$

where $\boldsymbol{B}^{(m, m)-1}(m \geqslant 2)$ is given by eqs. (2.6) and (2.22). The remaining onedimensional integrations in the above equations may be performed numerically.

\section{Numerical results for the fluctuation expansion}

In section 5 we have written the fluctuation expansion of the short-time self-diffusion coefficient $\boldsymbol{D}_{\mathrm{s}}$ in the form

$$
D_{s}=D_{s}^{(0)}+D_{s}^{(2)}+\ldots,
$$

where $D^{(p)}$ contains terms of order $\left\langle(\delta n)^{p}\right\rangle$, i.e. correlations of density fluctuations of order $p$.

From eqs. (5.7) and (6.11) we obtain for $\boldsymbol{D}_{s}^{(0)}$ the expression

$$
\begin{aligned}
\boldsymbol{D}^{(0)} & =D_{0} \boldsymbol{1}\left(1-\frac{2}{\pi} \phi \int_{0}^{\infty} \mathrm{d} k(\sin k / k)^{2} S(k)[1+\phi S(k)]^{-1}\right) \\
& =D_{0} 1 \frac{2}{\pi} \int_{0}^{\infty} \mathrm{d} k(\sin k / k)^{2}[1+\phi S(k)]^{-1}
\end{aligned}
$$

where the function $S(k)$ is defined in eq. (6.6). A numerical integration* yields the values listed in table $I$.

* We used an adaptive routine based on Gauss-quadrature rules ${ }^{22}$ ). This routıne, together with an d.gorithm for the evaluation of the sine-integral appearing in definition (6.6), is part of the NAG-library (Oxford) 
TABLE I

The fluctuation expansion (eq (7 1)) of $\boldsymbol{D}_{\mathrm{s}}$ The values under $\boldsymbol{D}_{\mathrm{s}}^{(0)} / D_{0}$ and $\boldsymbol{D}_{\mathrm{s}}^{(2)} / D_{0}$ result from eqs

(7 2) and (7 3), respectively

\begin{tabular}{ccccc}
\hline$\phi$ & $\boldsymbol{D}_{\mathrm{s}}^{(0)} / D_{0}$ & + & \multicolumn{2}{c}{$\boldsymbol{D}_{\mathrm{s}}^{(2)} / D_{0}=$} \\
\hline 000 & 1000 & + & 0000 & 100 \\
005 & 0896 & + & 0005 & 090 \\
010 & 0812 & - & 0007 & 080 \\
015 & 0743 & - & 0024 & 072 \\
020 & 0685 & - & 0041 & 064 \\
025 & 0636 & - & 0057 & 058 \\
030 & 0593 & - & 0071 & 052 \\
035 & 0556 & - & 0083 & 047 \\
040 & 0524 & - & 0093 & 043 \\
045 & 0495 & - & 0102 & 039 \\
\hline
\end{tabular}

With the ard of eqs (6 13) and (6 14) the expression (5 10) for $\boldsymbol{D}_{\mathrm{s}}^{(2)}$ reduces to

$$
\begin{aligned}
& \boldsymbol{D}_{\mathrm{s}}^{(2)} / D_{0}=\boldsymbol{A}_{n_{0}}^{(13)}(\boldsymbol{r}=0) \bigcirc \boldsymbol{B}^{(33)}{ }^{1} \bigcirc \boldsymbol{A}_{n_{0}}^{(31)}(\boldsymbol{r}=0) \\
& +\sum_{m=2}^{\infty} n_{0} \int \mathrm{d} \boldsymbol{r} \boldsymbol{A}_{n_{0}}^{(1 m)}(\boldsymbol{r}) \bigcirc \boldsymbol{B}^{(m m)-1} \bigcirc \boldsymbol{A}_{n_{0}}^{(m m)}(0) \\
& \bigcirc \boldsymbol{B}^{(m m) 1} \bigcirc \boldsymbol{A}_{n_{0}}^{(m 1)}(-\boldsymbol{r})+2 \sum_{m=2}^{\infty} n_{0} \int \mathrm{d} \boldsymbol{r} \boldsymbol{A}_{n_{0}}^{(1 m)}(\boldsymbol{r}) \bigcirc \boldsymbol{B}^{(m m)^{-1}} \\
& \bigcirc \boldsymbol{A}_{n_{0}}^{(m m+2)}(0) \bigcirc \boldsymbol{B}^{(m+2 m+2)}{ }^{1} \bigcirc \boldsymbol{A}_{n_{0}}^{(m+21)}(-\boldsymbol{r}) \\
& +\sum_{m-2}^{\infty} n_{0} \int \mathrm{d} \boldsymbol{r} \boldsymbol{A}_{n_{0}}^{(1 m)}(\boldsymbol{r}) \bigcirc \boldsymbol{B}^{(m m)^{-1}} \bigcirc \boldsymbol{A}_{n_{0}}^{(m 1)}(-\boldsymbol{r})[g(r)-1] \\
& +\sum_{m=2}^{\infty} \sum_{k=2}^{\infty} n_{0}^{2} \int \mathrm{d} \boldsymbol{r} \int \mathrm{d} \boldsymbol{r}^{\prime} \boldsymbol{A}_{n_{0}}^{(1 m)}(\boldsymbol{r}) \bigcirc \boldsymbol{B}^{(m m)}{ }^{1} \bigcirc \boldsymbol{A}_{n_{0}}^{(m k)}\left(\boldsymbol{r}^{\prime}-\boldsymbol{r}\right) \\
& \bigcirc \boldsymbol{B}^{(k k)}{ }^{\prime} \bigcirc \boldsymbol{A}_{n_{0}}^{(k 1)}\left(-\boldsymbol{r}^{\prime}\right)\left[g\left(\left|\boldsymbol{r}^{\prime}-\boldsymbol{r}\right|\right)-1\right]
\end{aligned}
$$

We have numerically evaluated all the terms in eq (7 3) not containıng connectors $\boldsymbol{A}_{n_{0}}^{(n m)}(\boldsymbol{r})$ with $n$ or $m$ larger than 2,1 e restricting ourselves to corrections to $\boldsymbol{D}_{\mathrm{s}}^{(0)}$ due to monopolar and dipolar hydrodynamic interactions between density fluctuations The results can also be found in table I We approximated $g(r)$ by the solution of the Percus-Yevick equation for hard spheres, as found by Wertheim and Thiele ${ }^{23}$ ) Detalls of the calculations are given in appendix D

The concentration dependence of $\boldsymbol{D}_{\mathrm{s}}$ given in table I will not be exact for two reasons a) the values given for $\boldsymbol{D}_{\mathrm{s}}^{(2)}$ result from monopolar and dipolar terms in eq (7 3) only, b) we have evaluated only the first two terms of the fluctuation expansion (7 1) To obtain an estimate for the errors due to a) and b) we have evaluated A) self-correlations ( $1 \mathrm{e}$ terms not containing the pair-correlation 
function) in eq. (7.3) due to quadrupolar or even octupolar hydrodynamic interactions between density fluctuations; B) self-correlations contributing to $\boldsymbol{D}_{\mathrm{s}}^{(3)}$. Since self-correlations give (for large $\phi$ ) the dominant contribution to $\boldsymbol{D}_{s}^{(2)}$ (see table II in appendix D), it seems fair to assume that the results from A) and B) are reasonable estimates of the errors due to a) and b), respectively. We found: A) higher multipole terms contribute $\approx 10^{-3} D_{0}$ to $\boldsymbol{D}_{\mathrm{s}}^{(2)}$ and can thus safely be neglected; B) $\boldsymbol{D}_{\mathrm{s}}^{(3)}$ will be $\approx 30 \%$ of $\boldsymbol{D}_{\mathrm{s}}^{(2)}$. Since $\boldsymbol{D}_{\mathrm{s}}^{(2)}$ in turn is less than $20 \%$ of $\boldsymbol{D}_{\mathrm{s}}^{(0)}$, one might expect the fluctuation expansion (7.1) to converge sufficiently rapidly.

We conclude that the values for $\boldsymbol{D}_{\mathrm{s}}$ given in table I should describe the concentration dependence of the short-time self-diffusion coefficient reasonably well up to high densities.

\section{Discussion}

We have calculated the concentration dependence of the short-time selfdiffusion coefficient $\boldsymbol{D}_{\mathrm{s}}$ for spherical particles in suspension.

For low values of the volume fraction $\phi$ a virial expansion is appropriate. We found ${ }^{11}$ )

$$
D_{\mathrm{s}}=D_{0} 1\left[1-1.73 \phi+0.88 \phi^{2}+\mathcal{O}\left(\phi^{3}\right)\right] .
$$

Only two-body hydrodynamic interactions contribute to the - well-known ${ }^{3.7}$ ) term of order $\phi$, which dominates if the suspension is very dilute. However, many-body hydrodynamic interactions may not be neglected at higher densities $\dagger$ : a neglect of three-sphere contributions would give a value of $-0.93 \phi^{2}$ instead of $+0.88 \phi^{2}$ for the term of order $\phi^{2}$ in eq. (8.1). In a concentrated suspension it is therefore essential to fully take into account the many-body hydrodynamic interactions between an arbitrary number of spheresł.

The expansion of $\boldsymbol{D}_{s}$ in correlations of fluctuations in the concentration of the suspended particles satisfies the above requirement. In fig. 1 we have plotted the results from this fluctuation expansion, including terms of second order in the fluctuation. At low values of $\phi$ we can compare these results with the virial expansion of $\boldsymbol{D}_{\mathrm{s}}$ (eq. (8.1)). We found from our fluctuation expansion a value of -1.96 for the first virial coefficient, in reasonable agreement with the value of -1.73 given in eq. (8.1), or with the exact value of -.1 .83 calculated by Batchelor ${ }^{3}$.

$\uparrow$ The same is true for bulk-diffusion ${ }^{11}$ ).

$\ddagger$ Van Megen, Snook and Pusey ${ }^{24}$ ) have averaged the two- and three-sphere mobilities given in eqs (4 I) and (4 6) with "exact" distributionfunctions (as known from computer simulations). The neglect of hydrodynamic interactions between four and more particles in a concentrated suspension can however not be justified. 


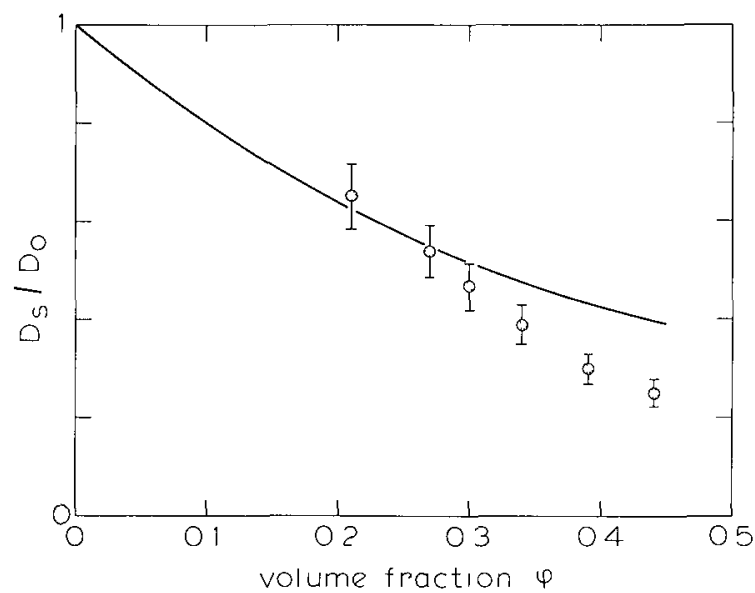

Fig 1 Density dependence of the short-tıme self-diffusion coefficient for spherical particles in suspension The solid curve is according to table I The datapoints with errorbars are measured values of $\boldsymbol{D}$, for a suspension of spherical colloidal latex particles (ref 14)

Pusey and van Megen ${ }^{14}$ ) have measured $\boldsymbol{D}_{\mathrm{s}}$ for a concentrated suspension of colloidal latex particles, using dynamic light-scattering techniques Their datapoints are also plotted in fig 1 Unfortunately, a comparison with the virial expansion (8 1) is not possible, since no measurements could be performed at sufficiently low concentrations As we can see, the fluctuation expansion is in reasonable agreement with the experimental results for $\phi \leqslant 030$ However, for $\phi \geqslant 035$ the calculated values for $\boldsymbol{D}_{\mathrm{s}}$ are much larger than the measured ones At $\phi=040 \mathrm{eg}$, experiment gives $D_{\mathrm{s}} / D_{0}=029$, while the fluctuation expansion yields $\boldsymbol{D}_{\mathrm{s}}^{(0)} / D_{0}+\boldsymbol{D}_{\mathrm{s}}^{(2)} / D_{0}=052-009=043$ In order to agree, higher order terms in the expansion would have to contribute $150 \%$ of the lowest order correction $\boldsymbol{D}_{\mathrm{s}}^{(2)}$ In view of the discussion given at the end of section 7 , this seems rather unlikely One may wonder, on the other hand, whether at these high densities - for which the typical distance between the centers of the particles is $25 a$ - the system studied in ref 14 may still be regarded as a suspension of hard spheres

We would like to conclude with the following observation the zeroth order result $\boldsymbol{D}_{\mathrm{s}}^{(0)}$ from the fluctuation expansion of $\boldsymbol{D}_{\mathrm{s}}$ (given by eq $(72)$ ) can, by means of the expansion (68) be approximated by

$$
\boldsymbol{D}_{\mathrm{s}}^{(0)} \approx \boldsymbol{1} k_{\mathrm{B}} T\left[6 \pi a \eta\left(1+\frac{5}{2} \phi\right)\right]^{-1}
$$

The approximation in eq (82) amounts to a restriction of the hydrodynamic interaction to the dominant monopolar and dipolar terms As we can conclude from a comparison with the exact values of $\boldsymbol{D}_{\mathrm{s}}^{(0)}$ given in table I, the error in $(82)$ is less than $5 \%$ The expression $\eta\left(1+\frac{5}{2} \phi\right)$ in eq (82) coincides - up to terms of order $\phi^{2}-$ with the effective viscosity of the suspension In this sense the zeroth 
order approximation $\boldsymbol{D}_{s}^{(0)}$ can be called an effective medium theory for selfdiffusion.

\section{Acknowledgements}

A discussion with Dr. P.N. Pusey is gratefully acknowledged.

This work was performed as part of the research programme of the "Stichting voor Fundamenteel Onderzoek der Materie" (F.O.M.), with financial support from the "Nederlandse Organisatie voor Zuiver-Wetenschappelijk Onderzoek" (Z.W.O.).

\section{Appendix A. Formulae for irreducible tensors}

We will use the following formulae (cf. ref. 15)

$$
\begin{aligned}
& \overline{\hat{\boldsymbol{r}}^{n-1}} \hat{\boldsymbol{r}}=\overline{\hat{\boldsymbol{r}}^{n}}+\frac{n-1}{2 n-1} \Delta^{(n-1, n-1)} \odot^{n-2} \overline{\hat{\boldsymbol{r}}^{n-2}}, \\
& \overline{\boldsymbol{r}}^{n} \odot^{n} \hat{\boldsymbol{r}}^{n}=n ! /(2 n-1) ! !, \\
& \boldsymbol{\Delta}^{(n, n)} \bigodot^{m} \boldsymbol{\Delta}^{(m, m)}=\boldsymbol{\Delta}^{(n, n)} \text { if } m \leqslant n, \\
& \boldsymbol{\Delta}^{(n, n)} \bigcirc^{m} \boldsymbol{\Delta}^{(m, m)}=0 \quad \text { if } m>n, \\
& \boldsymbol{\Delta}^{(n, n)} \odot^{n+1} \boldsymbol{\Delta}^{(n, n)}=\frac{2 n+1}{2 n-1} \boldsymbol{\Delta}^{(n-1, n-1)}, \\
& \frac{1}{4 \pi} \int \mathrm{d} \hat{\boldsymbol{r}} \widetilde{\hat{\boldsymbol{r}}^{n}} \widetilde{\hat{\boldsymbol{r}}^{m}}=\delta_{n m} n ![(2 n+1) ! !]^{-1} \Delta^{(n, n)} .
\end{aligned}
$$

Integrals of the form

$$
\boldsymbol{J}^{(n, m)} \equiv \frac{1}{4 \pi} \int \mathrm{d} \hat{\boldsymbol{r}} \widehat{\hat{r}^{n-1}}(\boldsymbol{1}-\hat{\boldsymbol{r}} \hat{\boldsymbol{r}}) \overparen{\hat{\boldsymbol{r}}^{m-1}}, \quad n, m \geqslant 2
$$

can now be evaluated using first eq. (A.1), and then eq. (A.6) and its corollary (cf. definition (2.19))

$$
\frac{1}{4 \pi} \int \mathrm{d} \hat{\boldsymbol{r}} \widehat{\hat{\boldsymbol{r}}^{n-1}} \hat{1} \overline{\hat{\boldsymbol{r}}^{m-1}}=\delta_{u m}(n-1) ![(2 n-1) ! !]^{-1} \Delta^{(n-1, \mathrm{dd}, n-1)} .
$$


The result is

$$
\begin{aligned}
& \boldsymbol{J}^{(n, n)}=(n-1) ![(2 n-1) ! !]^{-1} \\
& \times\left(\Delta^{(n-1, \mathrm{Id}, n-1)}-\frac{n}{2 n+1} \boldsymbol{\Delta}^{(n, n)}-\frac{n-1}{2 n-1} \boldsymbol{A}^{(n-1, n-1)} \bigcirc^{n-2} \boldsymbol{\Delta}^{(n-1 n-1)}\right), \\
& \boldsymbol{J}^{(n, n+2)}=\boldsymbol{J}^{(n+2, n)}=-(n+1) ![(2 n+3) ! !]^{-1} \boldsymbol{\Delta}^{(n+1, n+1)}, \\
& \boldsymbol{J}^{(n, m)}=0 \text { if } n \neq m \text { or } n \neq m \pm 2 \text {, }
\end{aligned}
$$

where we have made use of eq. (A.3) to simplify the expressions.

\section{Appendix B. Proof of equation (6.3)}

Every tensor of rank 2, constructed from the tensor 1 and the vector $\hat{r}$, is necessarily of the form $\alpha \mathbf{1}+\beta \hat{\boldsymbol{r}} \hat{r}$, with scalars $\alpha$ and $\beta$. Hence we can write

$$
(\boldsymbol{1}-\hat{\boldsymbol{r}} \hat{\boldsymbol{r}}) \overparen{\hat{\boldsymbol{r}}^{n-1}} \bigodot^{n} \boldsymbol{B}^{(n, n)-1} \bigodot^{n} \overparen{\hat{\boldsymbol{r}}^{n-1}}(\boldsymbol{1}-\hat{\boldsymbol{r}} \hat{\boldsymbol{r}})=\alpha \boldsymbol{1}+\beta \hat{\boldsymbol{r}} \hat{\boldsymbol{r}}, \quad n \geqslant 3
$$

with $\boldsymbol{B}^{(n, n)-1}(n \geqslant 3)$ given by eq. (2.22).

If we contract both sides of eq. (B.1) with $\hat{r}$, we find

$$
0=(\alpha+\beta) \hat{r}
$$

hence, $\beta=-\alpha$. To determine $\alpha$ we take the trace of eq. (B.1)

$$
\begin{aligned}
& \sum_{\mu_{1}} \sum_{\mu_{n} v_{1}} \delta_{v_{n}}{\widetilde{\hat{r}_{\mu_{1}} \ldots \hat{r}_{\mu_{n}}}}_{B_{\mu_{n}}^{(n, n)-1} \mu_{\mu_{1}, v_{1}}}{\overrightarrow{v_{n}}}_{\hat{r}_{v_{n}} \ldots \hat{r}_{v_{2}}} \\
& -\widehat{\hat{\boldsymbol{r}}} \hat{\boldsymbol{r}}^{n-1} \odot^{n} \boldsymbol{B}^{(n, n)-1} \bigodot^{n} \hat{\boldsymbol{r}}^{n-1} \hat{\boldsymbol{r}}=2 \alpha .
\end{aligned}
$$

The 1.h.s. of eq. (B.3) can be evaluated with the help of the formulae (cf. eqs. (A.1)-(A.5))

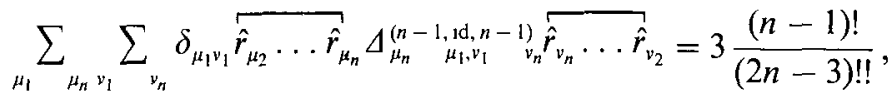

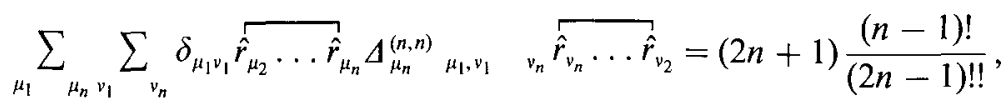




$$
\begin{aligned}
& \sum_{\mu_{1}} \sum_{\mu_{n} v_{1}} \delta_{p_{v_{1}} v_{1}} \overline{\hat{r}}_{\mu_{2}} \ldots \hat{r}_{\mu_{n}} T_{\mu_{n}}^{(n, n)} \mu_{\mu_{1}, v_{1}} \quad{ }_{v_{n}} \overline{\hat{r}}_{v_{n}} \ldots \hat{\hat{r}}_{v_{2}} \\
& =\overparen{\hat{\boldsymbol{r}}^{n-1}} \odot^{n-1} \overparen{\boldsymbol{r}^{n-1}}=(n-1) ![(2 n-3) ! !]^{-1},
\end{aligned}
$$

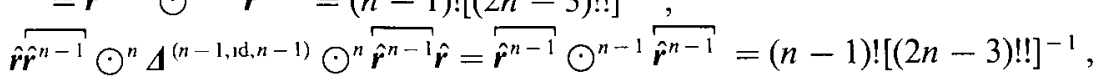

$$
\begin{aligned}
& \hat{\boldsymbol{r}} \widehat{\hat{\boldsymbol{r}}^{n-1}} \odot^{n} \boldsymbol{\Delta}^{(n n)} \odot^{n} \overline{\hat{\boldsymbol{r}}^{n-1}} \hat{\boldsymbol{r}}=\overline{\hat{\boldsymbol{r}}^{n}} \odot^{n} \widetilde{\hat{\boldsymbol{r}}^{n}}=n ![(2 n-1) ! !]^{-1}, \\
& \widehat{\boldsymbol{r}} \hat{\boldsymbol{r}}^{n-1} \odot^{n} \boldsymbol{T}^{(n, n)} \odot \bigodot^{n} \hat{\boldsymbol{r}}^{n-1} \hat{\boldsymbol{r}}=\hat{\boldsymbol{r}} \cdot \overparen{\hat{\boldsymbol{r}}^{n-1}} \odot^{n-2} \overparen{\hat{\boldsymbol{r}}^{n-1}} \cdot \hat{\boldsymbol{r}} \\
& =\frac{n-1}{2 n-3} \widehat{\hat{\boldsymbol{r}}^{n-2}} \odot^{n-2} \overparen{\hat{\boldsymbol{r}}^{n-2}}=\frac{n-1}{2 n-3}(n-1) ![(2 n-3) ! !]^{-1} \text {. }
\end{aligned}
$$

In eqs. (B.6) and (B.9) we have used the abbreviation

$$
\boldsymbol{T}^{(n, n)} \equiv \boldsymbol{\Delta}^{(n-1, n-1)} \odot^{n-2} \boldsymbol{\Delta}^{(n-1, n-1)} .
$$

Substitution in eq. (B.1) of the values for $\alpha$ and $\beta$ which follow from eqs. (B.2)-(B.10) yields

$$
(\boldsymbol{1}-\hat{\boldsymbol{r}} \hat{\boldsymbol{r}}) \widehat{\hat{\boldsymbol{r}}^{n-1}} \bigodot^{n} \boldsymbol{B}^{(n, n)-1} \bigodot^{n} \widehat{\hat{\boldsymbol{r}}^{n-1}}(\boldsymbol{1}-\hat{\boldsymbol{r}} \hat{\boldsymbol{r}})=-[(2 n-3) ! !]^{-2}(\boldsymbol{1}-\hat{\boldsymbol{r}} \hat{\boldsymbol{r}}) \quad n \geqslant 3 .
$$

Since $\boldsymbol{B}^{(2.2)^{-1}}$ equals $-\frac{10}{9} \boldsymbol{\Delta}^{(2.2)}$ (definition (2.6)), a simple calculation gives

$$
(\boldsymbol{1}-\hat{\boldsymbol{r}} \hat{r}) \hat{r}: B^{(2.2)^{-1}}: \hat{\boldsymbol{r}}(\boldsymbol{1}-\hat{\boldsymbol{r}} \hat{r})=-\frac{5}{9}(\boldsymbol{1}-\hat{\boldsymbol{r}} \hat{r}) \text {. }
$$

The eqs. (B.11) and (B.12) give, together with eq. (2.15) the required formula (6.3).

\section{Appendix C. Derivation of equation (6.5)}

We wish to calculate the sum

$$
\begin{aligned}
& \sum_{n=1}^{x}(2 n+1)^{2} J_{n+1 / 2}^{2}(x)=2 x^{2} \sum_{n=0}^{\infty}\left[J_{n+1 / 2}^{2}(x)+J_{n-1 / 2}(x) J_{n+3 / 2}(x)\right] \\
& \quad-x^{2}\left[J_{1 / 2}^{2}(x)+J_{3 / 2}^{2}(x)+2 J_{-1 / 2}(x) J_{3 / 2}(x)\right]
\end{aligned}
$$

where we have used the recursion-relation ${ }^{16}$ )

$$
(2 n+1) J_{n+1 / 2}(x)=x J_{n-1 / 2}(x)+x J_{n+3 / 2}(x) .
$$

In ref. $25(\$ 116)$ we find the useful formula

$$
\sum_{n=0}^{x} J_{n+v}(x) J_{n+\rho}(x)=x^{\rho-v} \int_{0}^{i} t^{v-\rho} J_{v-1}(t) J_{\rho}(t) \mathrm{d} t,
$$


for $v$ and $\rho$ real numbers If we take $v=\rho=\frac{1}{2}$, we find

$$
\sum_{n=0}^{\infty} J_{n+1 / 2}^{2}(x)=\pi^{-1} \int_{0}^{2 r} \sin t / t \mathrm{~d} t \equiv \pi^{-1} \mathrm{~S}_{1}(2 x),
$$

while the choice $v=3 / 2, \rho=-1 / 2$ gives

$$
\begin{aligned}
& \sum_{n=0}^{\infty} J_{n+3 / 2}(x) J_{n-1 / 2}(x)=x^{-2} \pi^{-1} \int_{0}^{x} t \sin 2 t \mathrm{~d} t \\
& =x^{-2} \pi^{-1}\left(\frac{1}{4} \sin 2 x-\frac{1}{2} x \cos 2 x\right)
\end{aligned}
$$

Substitution of eqs (C 4) and (C 5) in the sum (C 1) yields the result

$$
\sum_{n=1}^{\infty}(2 n+1)^{2} J_{n+1 / 2}^{2}(x)=(2 / \pi)\left[x^{2} \operatorname{Si}(2 x)+\frac{1}{4} \sin 2 x+\frac{1}{2} x \cos 2 x-x^{-1} \sin ^{2} x\right]
$$

This is the formula we need in deriving eq (6 5)

\section{Appendix D. Calculation of $D_{s}^{(2)}$}

We can write the integrals in eq (73) in terms of Fourier-transformed renormalized connector fields $\boldsymbol{A}_{n_{0}}^{(n m)}(\boldsymbol{k})$, which, according to eqs (2 14) and (69), are given by*

$$
\boldsymbol{A}_{n_{0}}^{(n m)}(\boldsymbol{k})=\boldsymbol{A}^{(n m)}(\boldsymbol{k})[1+\phi S(a k)]^{-1}
$$

Restricting ourselves to terms in eq (7 3) containıng only connectors with upper indices $n, m \leqslant 2$, the expression for $\boldsymbol{D}_{\mathrm{s}}^{(2)}$ takes the form

$$
\begin{aligned}
& \boldsymbol{D}_{\mathrm{s}}^{(2)} / D_{0}=n_{0}(2 \pi)^{-3} \int \mathrm{d} \boldsymbol{k} \boldsymbol{A}_{n_{0}}^{(12)}(\boldsymbol{k}) \boldsymbol{B}^{(22)} \quad \boldsymbol{A}_{n_{0}}^{(2)}(\boldsymbol{r}=\mathbf{0}) \\
& \boldsymbol{B}^{(22)} \quad \boldsymbol{A}_{n_{0}}^{(21)}(\boldsymbol{k})+n_{0}(2 \pi)^{-6} \int \mathrm{d} \boldsymbol{k} \int \mathrm{d} \boldsymbol{k}^{\prime} \boldsymbol{A}_{n_{0}}^{(12)}(\boldsymbol{k}) \boldsymbol{B}^{(22)^{-1}} \\
& \boldsymbol{A}_{n_{0}}^{(21)}\left(\boldsymbol{k}^{\prime}\right) v\left(\left|\boldsymbol{k}-\boldsymbol{k}^{\prime}\right|\right)+n_{0}^{2}(2 \pi)^{-6} \int \mathrm{d} \boldsymbol{k} \int \mathrm{d} \boldsymbol{k}^{\prime} \boldsymbol{A}_{n_{0}}^{(12)}(\boldsymbol{k})
\end{aligned}
$$

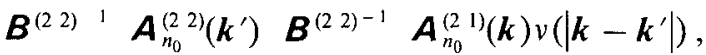

* When $\boldsymbol{A}_{n o}^{(n)}(\boldsymbol{r})$ appears as an integrand, we may in its representation (69) replace the modified connectorfield $\tilde{\boldsymbol{A}}^{(n m)}(\boldsymbol{r})$ by the unmodified field $\boldsymbol{A}^{(n m)}(\boldsymbol{r})$, since these two fields differ by a finite amount in a single point only (cf definition (34)) 
where $v(k)$ is the pair-correlation function in wave vector representation

$$
v(k)=\int \mathrm{d} \boldsymbol{r} \mathrm{e}^{\mathrm{l} k} r[g(r)-1]
$$

The first integral in eq (D 2) (due to self-correlations, of section 5) can be evaluated with the a1d of eqs (2 6), (2 15) and (6 12)

$$
\begin{aligned}
& \boldsymbol{D}_{\mathrm{s}}^{(2)} \text { (self-correlation) }=-\frac{135}{4} \pi \phi^{2} D_{0} 1 \int_{0}^{\infty} \mathrm{d} x x^{-4} J_{1 / 2}^{2}(x) J_{3 / 2}^{2}(x) \\
& \quad \times[1+\phi S(x)]^{-2} \int_{0}^{\infty} \mathrm{d} y y^{-1} J_{3 / 2}^{2}(y) S(y)[1+\phi S(y)]^{-1}
\end{aligned}
$$

A numerical integration ${ }^{22}$ ) of these one-dimensional integrals yields the values listed in table II, column I The two remaining integrals in eq (D 2) contain the pair-correlation function $v(k)$ We have approximated $v(k)$ by the solution of the Percus-Yevick equation for hard spheres $\left.{ }^{23}\right)^{*}$ Using eqs (26) and (2 15) we can write the two terms in eq (D 2) containing pair-correlations as two threedimensional integrals These integrals were evaluated by Monte-Carlo integration $^{20}$ ) The results can also be found in table II, columns II and III

TABLE II

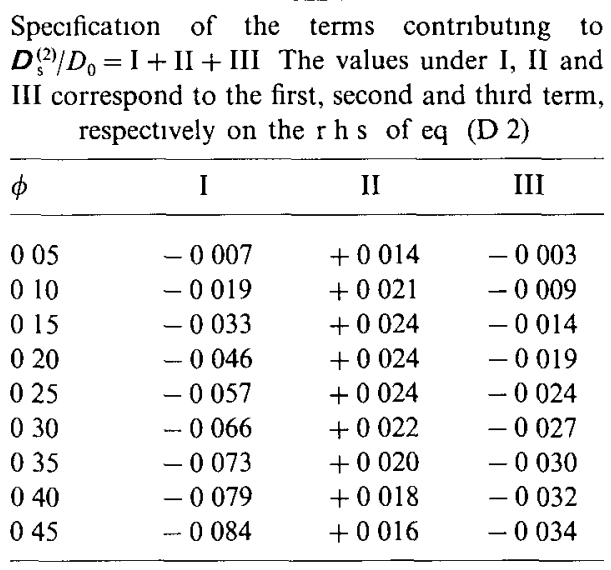

\section{References}

1) P N Pusey and R J A Tough, in Dynamic Light-scattering and Velocimetry, R Pecora ed (Plenum, New York, in press)

* An explicit analytic expression for $v(k)$ can be found in ref 26 
2) P N Pusey and R J A Tough, J Phys A15 (1982) 1291

3) G K Batchelor, J Fluid Mech 74 (1976) 1

4) H M Fijnaut, J Chem Phys 74 (1981) 6857

5) M Muthukumar, J Chem Phys 77 (1982) 959

6) B U Felderhof, J Phys A11 (1978) 929

7) R B Jones, Physica 97A (1979) 113

8) M M Kops-Werkhoven and H M Fijnaut, J Chem Phys 74 (1981) 1618

9) $P$ Mazur and $W$ van Saarloos, Physica 115A (1982) 21

10) G J Kynch, J Fluid Mech 5 (1959) 193,

T Yoshizakı and H Yamakawa, J Chem Phys 73 (1980) 578

11) C W J Beenakker and P Mazur, Phys Lett 91A (1982) 290

12) G D J Phillies, J Chem Phys 77 (1982) 2623

13) D Bedeaux and P Mazur, Physica 67 (1973) 23

14) P N Pusey and W van Megen, J de Phys 44 (1983) 285

15) S Hess and W Kohler, Formeln zur Tensor-Rechnung (Palm und Enke, Erlangen, 1980)

16) I S Gradshteyn and I M Ryzhık, Table of Integrals, Series and Products (Academıc Press, New York, 1965)

17) A J Weisenborn, private communication

18) B U Felderhof, Physica 89A (1977) 373

19) J G Kirkwood, J Chem Phys 3 (1935) 300

20) B Lautrup, Proc 2nd Coll on Adv Computing Methods in Theor Phys (Marseille, 1971)

21) E M Lifshitz and L P Pitaevskil, Statıstical Physics, part 1 (\$116) (Pergamon, Oxford, 1980)

22) E de Doncker, Signum Newsletter No 2 (1978) 12

23) M S Wertherm, Phys Rev Lett 10 (1963) 321,

E Thiele, J Chem Phys 39 (1963) 474

24) W van Megen, I Snook and P N Pusey, J Chem Phys 78 (1983) 931

25) N Nielsen, Handbuch der Theorie der Cylinderfunktıonen (Teubner, Leipzig, 1904)

26) F Mandel, R J Bearman and M Y Bearman, J Chem Phys 52 (1970) 3315 